BMJ Open

Sport \&

Exercise

Medicine

\title{
Lessons from Popper for science, paradigm shifts, scientific revolutions and exercise physiology
}

\author{
Robert Andrew Robergs
}

To cite: Robergs RA. Lessons from Popper for science, paradigm shifts, scientific revolutions and exercise physiology. BMJ Open Sport Exerc Med 2017;3:e000226. doi:10.1136/bmjsem-2017000226

Accepted 15 July 2017

\section{CrossMark}

School of Exercise and Nutrition Sciences, Queensland University of Technology, Kelvin Grove, Queensland, Australia

Correspondence to Dr Robert Andrew Robergs, School of Exercise and Nutrition Sciences, Kelvin Grove, Queensland, 4059, Australia; rob.robergs@qut. edu.au

\section{ABSTRACT}

A connection has been made to the possible role of the central governor model (CGM) to be a paradigm shift within the exercise sciences. Unfortunately, very little evidence was presented to support this notion, and a narrow view of scientific philosophy was used to reflect on the role of the CGM in understanding exercise physiology and the pursuit of a more ideal scientific method. When contrasting the scientific philosophies of Kuhn to Popper, and applying the tenant of falsification to the research and commentary on the CGM, it is probable that the scholarship pertaining to the CGM adheres more to pseudoscience than science. To improve the scientific contributions of research on the CGM, fellow scientists need to adopt a more critical platform where questions are raised and research designs are employed in efforts to refute the theory. The inability to falsify a theory is the most meaningful way to prove that it is likely to be correct. To support this development, the CGM needs to be more carefully worded to form a theory that clearly reveals key features that can be researched and potentially falsified. In addition, the wording of the CGM needs to allow scientists to make predictions that can then be tested in controlled experimental research studies. Until this happens for the CGM and all other pertinent paradigms within exercise physiology, the discipline will never rise out of the abyss of normal science to extraordinary science involving paradigm shifts and scientific revolutions.

\section{INTRODUCTION}

It was with great enthusiasm that I discovered and commenced the reading of the commentary by Pires, 'Thomas Kuhn's 'Structure of Scientific Revolutions' Applied to Exercise Science Paradigm Shifts: Example Including the Central Governor Model'. ${ }^{1}$ Three phrases within the title immediately caught my attention, 'Thomas Kuhn's 'The Structure of Scientific Revolutions', 'exercise science', and of course the 'Central Governor Model' (CGM). There is a need for more writing of the philosophical underpinnings of the scientific method. This is especially true in reference and application to the exercise sciences, and in particular the 20 years that have followed Noakes' first account of his $\mathrm{CGM},{ }^{2}$ and the 30 years since Katch's provocative call for greater critical research enquiry within the exercise sciences. ${ }^{3}$

Unfortunately, my enthusiasm was shortlived. When aware of the fundamental tenants of the scientific method, numerous flaws within this commentary and by the journal are obvious and require constructive criticism. Yet before such content can be explained, what are some of the fundamental tenants of the scientific method, and why have I developed an interest in them? Approximately 15 years ago, contemplation of my prior reading and research led me to conclude that most of the science I was involved in seemed to operate disconnected from what I thought science should be. At that time, my awareness of science was heavily influenced by a classic commentary by Katch, ${ }^{3}$ as expressed above. In more recent time, after reading more and more published manuscripts that just did not seem to make sense from fundamental research design, as well as knowledge of organic chemistry, biochemistry and multiple systems physiology, I began to question my own contributions to science. In short, I forced myself to step back, outside of myself, and view what type of scientist I was. This is an exercise that I think all scientists should do, and repeatedly do throughout their career. What I saw disturbed me. I researched topics that were easy to study, supported paradigms that I was taught, and developed a research agenda that followed the easiest path to attaining numeric goals for my publication list that was most conducive to me attaining tenure and promotion. I was not critical in my view of research, and when I detected flaws, I turned a blind eye as I did not want to disturb the momentum of a developing career based solely on research quantity.

I made a commitment to myself that to be a scientist with integrity, I had to challenge 
paradigms within exercise physiology that I viewed to be incorrect, based on empirical evidence of others and my own research, and to respond to the call of Katch. $^{3}$ That led to my refutation of the use of maximal heart rate estimation from the equation 220 - age, ${ }^{4}$ the lactic acidosis construct, ${ }^{5}$ the methods used in the analysis of $\mathrm{VO}_{2}$ kinetics to steady state, ${ }^{6}$ and numerous other critical commentaries and experimental research based on adopting an approach framed around critical enquiry; that based on Popper's ${ }^{7}$ requirement that scientific research be founded on the intent to falsify accepted paradigms when there is appropriate empirical support (not opinion) to do so. The fact that I did not do this in my prior research career meant that I was at risk for pursuing more pseudoscience than science. Detailed explanation of what separates science from pseudoscience will be provided in the section 'Lessons from Popper on the theory dervied from the CGM'.

Despite this awakening to some of the fundamental tenants of what made science scientific, years of frustration followed based on an editorial peer review system that functioned more to reinforce convention than nurturing critical enquiry. The more reading I did of scientific philosophy, the more I became frustrated at how the industry of science was not focused on the ideal pursuit of the scientific method. It was a system intent on preserving the status quo based on allowing the insertion of bias into peer review, at a huge cost of stifling progress.

Such a background is important for this commentary, as it places context to the need for critical challenge in science. During the reading of Pires' commentary, there were clear demarcations from critical science. Indeed, whenever I read a manuscript that solely leverages support to a paradigm, there are immediate questions raised about the intent and rigour of the content being published. For example, Kuhn is not a logical research historian to frame a reference of expected features of the scientific method. As eloquently explained by Katch, ${ }^{3}$ Karl Popper is the philosopher who has contributed most to the understandings of the ideal tenants of the scientific method. ${ }^{7}$ Pires then directed attention to the possibility for Noakes' CGM to be an example of what Kuhn described as 'extraordinary science' leading to a revolutionary paradigm shift in exercise science. However, I have always been perturbed by the process at which the debate around the CGM has progressed. The manuscript of Pires $^{1}$ and this rebuttal presented opportunities for me to express such concern, which again is not about favouring one side or the other. It is an opportunity to reveal that when the key tenants of science are not adhered to, by any side of a debate, then there is a huge risk for misinformation to be conveyed, leading to false knowledge generation.

While I have a great deal of respect for Noakes' efforts to improve our understanding of the determinants to exercise tolerance, there are numerous philosophical features of the process that has been used to develop the CGM that require critical commentary. Given the nature of the content of Pires' commentary, now is the time to do that based on an intent for recognising the importance of nurturing the integrity of the scientific method as the priority of a scientist's purpose. Consequently, the purpose of this manuscript was to provide a rebuttal, structured by topics that include the three titled items of interest, conduct of the journal, as well as other specific examples of errors in the evidence Pires used to frame his content. In addition, and most importantly, comment will be given on important lessons from Karl Popper ${ }^{34}$ on the essential traits of the scientific method that contemporary science in general, and especially exercise physiology, seem to still have forgotten.

\section{Exercise physiology is more than a discipline of exercise science}

Historically speaking, exercise science developed from the discipline of physical education sometime around the 1980s, depending on the country of reference. The initial development of exercise science was framed around five subdisciplines: exercise physiology, motor learning/control/skill acquisition, growth and development, biomechanics, and sport sociology/psychology. These subdisciplines became known as the " 5 pillars of exercise science'. ${ }^{8}$ Today, thanks to decades of continued research and knowledge creation, one could argue, at least in the Australian context, that added pillars have now emerged. From the exercise physiology pillar alone has grown emphases in strength and conditioning, clinical exercise physiology, sports physiology and perhaps others.

The emergence of exercise physiology as a discipline of exercise science does not mean that exercise physiology is confined to exercise science. For more than 100 years, researchers have investigated human and animal physiological responses to exercise stress. Such research has been pursued by metabolic biochemists, specialist physiologists (eg, cardiovascular, pulmonary, neural, endocrine, muscle, bone and so on), clinicians (cardiologists, pulmonologists, physiotherapists, nutritionists, oncologists, epidemiologists and so on) and of course exercise scientists. The rich multidisciplinary input and applications of exercise physiology have been major factors in the rapid growth of knowledge, and as with all disciplines of science, no topic is owned by any one discipline. The fact that this manuscript is partly in response to work done by a cardiologist interested in a diverse array of human physiological enquiry during exercise stress is testament to this fact.

Thomas Kuhn's 'Structure of Scientific Revolutions'

Kuhn (1922-1996) did not write his text with the intention of presenting a model for how the scientific 
method, or science itself, should function. ${ }^{9}$ Kuhn commenced his scientific career as a physicist, but was soon drawn to the understandings of the historical development of science. Kuhn was interested in the progression of scientific discovery, and he studied science from a historical perspective, not from a judgement of right or wrong. Kuhn explained this in the Preface to his textbook and concisely expressed the purpose of his text within the Introduction: Its aim is a sketch of the quite different concept of science that can emerge from the historical record of the research activity itself $\left(\mathrm{pl}^{10}\right)$.

It is also important to understand that Kuhn published his textbook in 1968. Contemporary science is a much different process to how it was in 1968, and for the centuries that preceded that time and provided the observations that fuelled Kuhn's passion. Nevertheless, Pires commented that 'Kuhn's model', presumably in reference to a model of scientific progress, was that of extended periods of stagnation, which Kuhn labelled 'normal science', separated by bursts of revolutionary discovery. ${ }^{1} 910$ What Pires failed to mention was that Kuhn's choice of words for labelling the periods of scientific advancement has been widely criticised by his peer scientific philosophers, and his greatest critic was Karl Popper. ${ }^{7}{ }^{11}$ Indeed, Kuhn's work is contrasted by Popper's more idealistic views of what the scientific method should be. This Popperian lens revealed that the periods of stagnation that characterises so much of the history of science is far from 'normal science'. Rather, such troughs between infrequent peaks of paradigm shifts to scientific revolutions result from dysfunctional scientific enquiry; so dysfunctional that Popper would label it, both in Kuhn's time ${ }^{11}$ and even 30 years before Kuhn published his text, ${ }^{7}$ as pseudoscience. I find it intriguing that Kuhn did not reference Popper in any part of his text, and that Pires reinforced the same omission.

\section{The omission of the work of Karl Popper}

I do not understand why Pires, a person selfproclaimed to be 'trained in the philosophy of science' (Pires ${ }^{1}, \mathrm{p} 722$ ), would choose to base a manuscript on the philosophical nature of exercise science (exercise physiology) framed around the views of Kuhn. As previously explained, Kuhn did not write on how science should operate. That topic was masterfully crafted by Popper, ${ }^{71}$ who remains our most influential contemporary philosopher of science.

Popper's greatest contribution to the development of the continually evolving scientific method was the concept and practice of falsifiability. Popper argued vehemently throughout his long life and career (19021994) that no scientific investigation could prove anything. No matter how many similar findings resulted from research on the same topic from different scientists from different institutions, it only requires one contradictory finding, preferably replicated elsewhere, to falsify, or at least seriously question, the result/theory. The preferred evidence of proof for a finding or theory was that of continued failed efforts to falsify the result/theory. However, Popper also went to great efforts to express that the development of theories is central to the scientific method. Furthermore, no theory could ever be perfect, and scientific endeavour through falsification would provide evidence to refine the result/theory, leading to improvement and progress on a path towards the truth.

Popper even went further to distinguish science from pseudoscience. To Popper, researchers who deviated from the tenant of falsification were not following the most fundamental trait of the scientific method, and hence were pursuing pseudoscience. Indeed, Popper proclaimed that the tenant of falsification is a 'criterion of demarcation' between science and pseudoscience. In this regard, and of importance to the commentary by Pires, Popper's writing on Kuhn's work from the published proceedings of the International Colloquium in the Philosophy of Science, London 1965, is an interesting read. ${ }^{11}$ Remember, Kuhn's text ${ }^{9}$ was published 30 years after Popper's. ${ }^{7}$ Popper viewed Kuhn's reported periods of stagnation as evidence of the acceptance of current theories and dogma. Such practice (Kuhn's 'normal science') risks reinforcement of false knowledge and derails the progress of science from a path towards the truth. Popper's writing revealed disgust of Kuhn's views of 'normal science', ${ }^{10}$ as he has labelled the scientists involved in this practice as 'to be sorry for', 'been badly taught' and 'a victim of indoctrination'. ${ }^{11}$ For Pires ${ }^{1}$ to assign an understanding of Kuhn's views to exercise physiology and the CGM is to cast a murky shadow for how exercise physiology has and perhaps remains largely pseudoscientific.

Nevertheless, contemporary science is very different from Popper's views, first published way back in $1934 .^{7}$ As such, it is important to explain what Popper's pseudoscience is so that our current interpretation can be placed in perspective. Popper had a simplistic view of the delineation between science and pseudoscience. Any non-critical application of science, that not based on efforts at falsification, was pseudoscientific. This is a very broad brush from which to define pseudoscience. Debate continues today within the field of the philosophy of science as to how relevant Popper's delineation of this transition to pseudoscience is. Yet an awareness of pseudoscience for any given attempt at research may not be apparent in current time. A researcher may try to be totally ethical, meticulous in research design and data analysis, and detailed in the writing of the discussion. Some readers may wonder how such initiative and effort could ever be pseudoscientific. This is where Popper's greatest contribution becomes apparent. Popper has challenged us all to be self-aware of our purpose or intent in research. What are the reasons for conducting the research? Are we following a mindset 
based on being aware of limitations in prior research, or current paradigms, and are we challenging this? Alternatively, is our research based on an extension of prior accepted paradigms? If so, has there been sufficient critical enquiry of the paradigm? Is it factual? If not, then Popper would argue that research should continue to challenge the paradigm, and research that supports it is at great risk of contributing to knowledge generation that is of no long-term value; or worse, is detrimental, or false knowledge. This then would be pseudoscience, even if the efforts in research were positive in intent. Similarly, and this applies to commentary on Noakes' CGM, being critical but not following sustained efforts at remaining critical, even of a new theory, is also pseudoscientific, as a researcher should never favour one paradigm over another, even if they propose it. This also fits Popper's definition of pseudoscience. This will be discussed in great length in the section 'Lessons from Popper on the theory derived from the CGM'.

\section{Curious interpretations of citation numerics}

Pires devoted considerable attention to the belief that the number of citations can be used to infer acceptance of a topic/theory, and presented numerics of such citations for the CGM in text, as well as two figures. Does Pires not understand that his methods for compiling these numerics contain both citations supportive and critical of the CGM? To fully reveal this flaw, I adopted a similar method to Pires and counted the peerreviewed publications on the topic of intelligent design' for the years 2000-2016 through PubMed. Just from this one database I retrieved 200 published manuscripts where 'intelligent design' was stated in the title or abstract. Based on Pires' interpretations, intelligent design must be a widely accepted construct in evolutionary science. Obviously, this is an incorrect interpretation. Intelligent design is a topic that infers divine intervention, which clearly cannot be experimentally tested and then by definition cannot be falsified. In extension, the topic therefore fails Popper's criterion of demarcation and is pseudoscience. Journal citations or topical preference cannot be interpreted as evidence of acceptance or agreement.

\section{Lessons from Popper on the theory derived from the CGM}

An important contribution from Popper was his application of falsifiability to the development and testing of theories. In this regard, let me clarify that Noakes' CGM is not itself a theory. Models are expressions of the connections between components of a system, or systems, often presented in diagrammatic form, from which theories can be worded or vice versa. Nevertheless, it has been a difficult process to explore the prior commentary and research on the CGM to find the exact wording of the theory. I have never understood why Noakes has not clearly stated the CGM as a theory , for as explained in great detail by Popper, ${ }^{7}$ the theory requires the researcher to communicate essential traits of the model that allow them and other researchers to attempt to falsify the theory. It is through failed attempts at falsification that a theory is best affirmed. In 1997, Noakes stated his 'alternative model' to understanding the limits of muscle contractile function (the beginnings of the CGM) to be as follows: '...skeletal muscle contractile function is regulated by a hierarchy of controls specifically to prevent damage to any of a number of different organs'. ${ }^{2}$ Yet, of course, this is neither a model nor theory; it is simply a statement. While theories are also statements, a theory must be worded carefully to adhere to a few simple rules, which I will detail below.

Is the textual explanation of the original CGM a wellworded theory? Let us evaluate Popper's teachings to us. Popper argued that a theory must also be stated in such a way that it is open to falsification. Indeed, as previously explained, Popper proclaimed that it is the process of falsification, as well as the invitation for it, that is the foundation of the scientific method. Consequently, for a theory to be scientific, it must be worded in such a way to allow other researchers to identify one or more features that can be used to make predictions and for these predictions to be able to be directly challenged by experimentation. If the wording of a theory hampers efforts at falsification, then there is risk for the absence of structured attempts at falsification, and by definition, retarded progress. Furthermore, if a theory is worded in such a way that it cannot be falsified, then based on Popper's criterion of demarcation, that act qualifies as pseudoscience and renders all research that the pseudoscientific theory has spawned to also be open to the label of pseudoscience.

It is important to note that the potential for falsification is the key, as some theories that are valid can be crafted even though there is no means yet to experimentally test them. The best example of this was Einstein's general relativity theory. Einstein drafted this theory in 1905. It was not until 1938 that the first empirical evidence was produced to provide proof of the theory based on measurements of the bending of light in a gravitation field. ${ }^{12}$

What can be tested from the first version of the CGM? There are three components that are raised: (1) a hierarchy of controls, (2) organ damage and (3) for the purpose of preventing organ damage. The wording of items 1 and 2 are vague and adhere to Popper's concerns of being unfalsifiable. Item 3 is teleological, as it infers that physiological systems have a singular purpose, which then explains why it exists. This cannot be falsifiable and as such is pseudoscientific.

As previously explained, the CGM has developed since this initial expression. How has it changed and how suitable are these new expressions as theories?

In 2000, Noakes further discussed the importance of AV Hill's original proposition that a neural governor must regulate function during fatiguing exercise in 
order to prevent organ damage. ${ }^{13}$ At this time, Noakes favoured a more task-dependent approach to explaining limits to exercise tolerance. However, no version of the CGM was presented as a graphic model or in text as a theory.

The first presentation of the CGM was provided in figure 1 of Noakes $^{14}$ (p3232) manuscript on the evidence for a central governor during exercise in acute hypoxia and hyperoxia. ${ }^{14}$ Noakes referred to the CGM first as a theory and synonymously as a model. However, from now on, I will refer to it as the CGM to avoid any confusion. The CGM was expressed as follows:

\section{'...the reduced fHmax, Vsmax and Qmax during acute and chronic hypoxia are due to central regulation, the goal of which is to prevent the development of hypoxia in one or more of the vital organs including the brain, heart or perhaps the respiratory muscles. The action of this central governor is to regulate the mass of muscle that can be recruited during exercise under conditions in which the oxygen delivery to these vital organs is threatened. By limiting the muscle mass that can be activated, the central governor limits the peripheral peak $\mathrm{VO}_{2}$ to a level that will not induce hypoxia in any of the vital organs'.}

In this context, and at this time, the CGM was specific to cardiovascular and neuromuscular regulation by a specific region of the central nervous system (CNS) that acted as a governor to process afferent input to fine-tune (dampen) motor output to prevent continued increases in $\mathrm{VO}_{2}$, oxygen demand and the consequent cardiovascular stress that would be associated with these events.

In 2004, Noakes then presented further evidence for the relevance of the CGM to all forms of exercise likely to stress organ function. ${ }^{15}$ The CGM was stated as follows:

\footnotetext{
‘. during self-paced exercise, which is the natural, as opposed to laboratory, form of human physical activity, the CNS continuously modifies the pace as part of a complex, non-linear dynamic system. In this model, the power output (work rate) during exercise is continuously adjusted on the basis of metabolic calculations performed at a subconscious CNS level. These calculations take into account prior knowledge acquired during previous exercise bouts, the planned end point of the current exercise bout, and the current metabolic rate, among many other potential variables. These subconscious calculations create a continuously adjusting power output, and hence pace, during exercise'.
}

This was a very different explanation to the prior version, as it now pertained only to self-paced exercise. However, the key evidence for supporting the CGM remains an altered motor unit recruitment. In fact, at this phase of the development of the CGM, there was clear direction for the need to measure motor unit recruitment (at least estimate it as best as possible from electromyography (EMG) based on current limitations in instrumentation) as part of any experimentation aimed at falsification (see next section). Nevertheless, it is important to acknowledge that there is controversy regarding the validity of surface EMG signals to be reflective of motor unit recruitment. ${ }^{16-19}$ As such, it remains dubious at best to even infer that changes in human motor unit recruitment can be accurately quantified during changes in exercise intensity and/or physiological transitions to fatigue at constant exercise intensities. This fact also raises concern for how Noakes has interpreted research of EMG signals during exercise to support a reduced motor unit recruitment component within the CGM.

Also in 2004, St Clair Gibson and Noakes presented another version of the CGM (referred to as the "central integrative model', and also the 'central governor theory of fatigue') in their figure 6 (St Clair Gibson $\left.^{20}, \mathrm{p} 801\right)$. This version was focused on exercise and fatigue (a term which I have avoided by using the phrase 'exercise tolerance'). This version of the CGM was stated as follows:

\begin{abstract}
'... the subconscious brain sets the exercise intensity by determining the number of motor units that are activated and hence the mass of the skeletal muscle that is recruited throughout the exercise bout. The extent of motor neurone activity and hence skeletal muscle motor unit recruitment can then be influenced by sensory feedback from a variety of peripheral organs, yet to be characterised and which probably include the skeletal muscles, the respiratory muscles, the heart, and central and peripheral chemoreceptors and mechanoreceptors. From the beginning of the exercise bout, the subconscious brain informs the conscious brain of an increasing neural effort, perhaps related to an increased difficulty in maintaining homeostasis at the exercise intensity and this is interpreted by the brain as the increased sensation of fatigue, which may itself control further subconscious brain control processes'.
\end{abstract}

This version of the CGT now pertains to all forms of exercise.

In 2005, the CGM was further modified to include added details of the roles of the subconscious brain in the perception of fatigue, as well as an integration of both central and peripheral factors in the regulatory scheme. $^{21} 22$ The model was now referred to as the central governor model of fatigue incorporating teleoanticipation during exercise' ( $\mathrm{p} 58^{22}$ ) or more simply as the 'integrated complex governor model of fatigue' (Lambert et $a l^{22}, \mathrm{p} 61$ ). Unfortunately, the description of the new CGM was excessively long, but its inclusion is needed in this rebuttal so there can be a critical reflection of the content in the next section. The new CGM was stated as follows:

\footnotetext{
'In the context of a complex and integrated model of fatigue, during exercise, there is no single regulatory component. Rather, there are multiple levels of regulation, resulting in integrative homeostatic control of the different physiological systems, which is a continuous oscillatory process, and which thereby invokes compensatory responses in the periphery. There is also, however, evidence for central programming and a feed forward component in this
} 
complex model, which serves to alter the settings at which gain and the time constant of the gain of the entire system are achieved, and which thereby alters the perception of fatigue.

Furthermore, in this complex model, there are redundancies in control mechanisms that can be altered by antecedent exposures to exercise or metabolic perturbations and external environmental factors. These multiple controls and redundancies produce a robust system, providing for changes in gain and the time constant of gain as part of a general homeostatic process. These homeostatic controls maintain system integrity and ensure that any system is not overwhelmed or used to absolute maximal capacity using continuous feed forward and feedback control. The integrated complex governor model of fatigue therefore recognises multiple interdependent control systems, the importance of peripheral metabolic factors as signallers, and that the teleoanticipatory central nervous system is mutable and can be 'reset' or 'hard wired' by various stimuli such as prior experience, antecedent exposures, and training.'

In 2007, Noakes applied the CGM to better understand marathon running. ${ }^{23}$ However, Noakes used the CGM without clarification of the exact version of the model he was applying. Based on the descriptions used, it seems that Noakes was referring to the integrated complex governor model of fatigue'.

In 2008 Noakes $^{24}$ presented a very similar description of the CGM to what was presented in 2005. Nevertheless, the wording was different, and in science, words matter. Noakes referred to this theory or model as the 'anticipatory (central governor) model', and stated it to be as follows:

\footnotetext{
'. . .human exercise performance is regulated (not limited) by a complex, intelligent system, the goal of which is the maintenance of homeostasis in all body systems. According to this model, subjects begin exercise at an intensity that is determined by their physiological capacity including their state of training, the expected duration of the exercise bout, their previous experience and the relative importance of the exercise bout, among many other influences. This established the feed-forward component of the central motor command, which determines the initial pace by recruiting the appropriate number of motor units in the muscles in the exercising limbs. Feedback form a variety of organs that monitor both the internal and external environment, then modify the pace by altering the number of motor units recruited in the exercising limbs. The goal of this control is to ensure that the exercise bout terminates before there is damage to any organ system as a result of a failure of homeostasis. The symptoms of fatigue become progressively more severe during exercise in order to ensure that exercise terminates within a predetermined and safe duration'.
}

Since 2004, the theory has developed to read more like a thesis than a theory. However, more on that in the next section. Later manuscripts from Noakes ${ }^{25} 26$ refer back to the prior versions of the CGM as stated since 2004, but given that the wording, components and function of each iteration are very different, it is unclear which specific version he was applying his current thinking and written expression to. Noakes had the opportunity to update everybody about the latest version of the CGM in his 2012 synopsis of the CGM and recent research that might support it; unfortunately, he did not. ${ }^{26}$

\section{The central governor theory: a paradigm shift or pseudoscience?}

What is wrong about the CGM? Or perhaps a better question is, how can it be improved? There is value for detailing specific aspects of each iteration of the CGM to decipher the presence of content that is unfalsifiable, versus content that can be gleamed from the words to focus attempts at falsification.

\section{7 version}

A critique of the original expression of the CGM was provided in the previous section. Clearly, Noakes wrote the initial version of the CGM with a prior awareness of the lessons from Popper regarding falsification, as Noakes $^{2}$ quoted Popper in the manuscript from his JB Wolfe memorial lecture: 'A statement (a theory, conjecture) has the status of belonging to the empirical sciences if and only if it is falsifiable'. ${ }^{7}$ Nevertheless, Noakes did not apply such knowledge to this poorly written version of the initial CGM.

\section{1 version}

The 2001 concept of a central governor was specific to hypoxia and hyperoxia, and therefore to the regulation of oxygen delivery and tissue oxygenation. There are focal topics from this theory that can be gleamed and used to frame experimentation for falsification:

1 . The neural regulation was proposed to be centrally located, presumably within the CNS and morphologically in a singular location (governor, a person or device providing regulation or leadership). Any evidence of complex neural regulation from a diverse number of locations, both central and peripheral, would falsify the theory.

2. The CGM functions to prevent the development of hypoxia in one or more vital organs. Thus, research that documents tissue hypoxia in one or more organs would falsify the model.

3. Noakes commented that reducing motor unit recruitment during exercise will preserve oxygen delivery to vital organs. Even if we disregard the problematic topic of quantifying motor unit recruitment, I am not aware of any research that supports this assertion. Rather, there is compelling evidence spanning multiple decades of research enquiry that organ blood flow is well preserved during intense exercise, and if anything, skeletal muscle blood flow is compromised in order to sustain an adequate central blood volume, central blood pressures and peripheral cardiovascular perfusion pressures to other tissues. ${ }^{27} 28$

4. Noakes needs to explain his rationale for connecting increasing muscle $\mathrm{VO}_{2}$ to the risk for tissue hypoxia 
in the vital organs other than the heart. Why does muscle $\mathrm{VO}_{2}$ directly influence the oxygenation of these other tissues? Interestingly, research based on measurement of myoglobin deoxygenation clearly shows that muscle hypoxia is developed during intense exercise, where muscle $\mathrm{PO}_{2}$ has been estimated at close to $0 \mathrm{~mm} \mathrm{Hg} .{ }^{29}$ Indeed, and as has been argued by critics of the $\mathrm{CGM}^{30-32}$ and others, ${ }^{27-29}$ there is a clear likelihood that due to the sacrifice of blood flow to working muscle during high exercise intensities, the CGM may in fact contribute to a cardiovascular limitation to exercise tolerance via constraints to continued increases in cardiac output. In this context, the debate around the cardiovascular versus non-cardiovascular (CGM) determinants of exercise tolerance becomes an issue similar to the chicken versus egg corollary; does it really matter?

\section{4a version}

As previously stated, this version was now written to pertain specifically to self-paced exercise. The important components pertaining to falsification were the following:

1. The theory states that the goal of the CGM is to prevent hypoxia. It is impossible to ascertain the goal of any physiological system, and as such this content is pseudoscientific.

2. Self-paced exercise is a superior mode of exercise to research for improved understanding of the physiological regulation of exercise tolerance. However, there is limitation to this view when concerned with elite athletic and sports performance, where added external drivers of effort enter into the performance equation that interfere with total volitional control over the exercise intensity.

3. Reference to 'metabolic calculations' performed by the subconscious brain is unfalsifiable and therefore pseudoscientific.

4. If the continually adjusting power output during self-paced exercise is a result of peripheral signals and CNS processing, then surely the peripheral contributions are evidence of the CNS being a slave to the periphery, not vice versa.

5 . Research needs to be done to quantify the relationship between alterations in motor unit recruitment and markers of peripheral function that return afferent feedback to the CNS. If these relationships do not exist, then that is evidence of falsification of the CGM.

6. Motor unit recruitment is a key to the model. If it can be shown that motor unit recruitment does not decrease as expected during documented decrements in muscle force production, then this clearly opposes and therefore falsifies the theory. Consequently, researchers need to improve the methods available to more validly quantify motor unit recruitment, or alternatively, develop exercise models that minimise the limitations of the measurement of human motor unit recruitment from surface EMG. ${ }^{16-19}$

7. What is the physiological direct connection between increasing muscle $\mathrm{VO}_{2}$ and increasing risk for tissue hypoxia in a diversity of body organs? If this connection can be proven to not exist, then this is further evidence for the theory to have been falsified.

\section{4b version}

This version was now written to pertain to the development of fatigue during all exercise conditions, and was very similar to the prior 2004 version. There were no new items of falsification to identify.

\section{5 version}

This version was a clear deviation in the CGM towards increased complexity commensurate with an integrated multisystems focus on exercise tolerance. The important components pertinent to falsification were the following:

1. The account of the regulatory scheme (initial sentences and final sentences) implies that such regulation occurs in one location within the CNS, and as such is highly organised. This differs from a scheme where multiple regulatory systems operate independent of each other, yet combine to result in a relatively sophisticated conglomerate regulation. Evidence of the latter would falsify the theory.

2. There could be tests of falsification to the notion that organs and/or systems are not overwhelmed or used to maximal capacity. However, such measurements may currently remain constrained by limitations in technology and instrumentation.

\section{8 version}

This is the final version of the CGM that was identified from a review of the scholarship of Noakes to 2016 . This version was relatively similar to the 2005 version, but more concise yet constrained by wording that reveals a teleological rationale for connecting regulation to purpose. The important components pertinent to falsification were the following:

1. It is incorrect to label human neural physiology regulation as intelligent, as 'intelligence' is a construct that pertains to human behaviour. Consequently, the intelligence of the neural regulation of the theorised CGM system cannot be measured, and hence cannot be falsified, which in turn means it is pseudoscientific.

2. For the same reason as for item 1 above, you cannot infer a teleological purpose or goal of a physiological regulatory system, which in this case is stated to be the 'maintenance of homeostasis in all bodily systems'. That said, experimentation that reveals divergence from homeostasis during exercise stress in one or more systems would falsify the theory.

3. Exercise conditions that cause damage to tissues/ organs during exercise would falsify the theory. For 
the heart, why do individuals with coronary arterial atherosclerosis experience exercise-induced myocardial ischaemia if a CGM presumably should prevent this? Interestingly, what about skeletal muscle? There is overwhelming evidence of damage to skeletal muscle to sustained exercise, of even low intensity, that is obviously not prevented by the CGM. Another example of damage that is catastrophic is exercise-induced muscle cramp. How does this example fit, or not fit, in the CGM?

\section{Additional concerns}

There are added global concerns about fundamental scientific principles associated with the CGM. The first to raise is the notion that the regulatory physiological events that concern final constraints to exercise tolerance are viewed to be the most important. However, what is the rationale for this view? Perhaps it is best to use an analogy to a common proverb of 'the straw that broke the camel's back'. The CGM is akin to claiming that the final straw is special and different from each and every other straw simply because it caused the final collapse of the camel. In reality, each straw is equal, even the first which does no apparent or perceived harm, through to the last. What makes the involvement of neural processing so important when there are other known contributors to the physiological constraints/ perceptions leading to the involuntary or voluntary end to exercise (exercise tolerance)?

There is also another way to look at the physiology involved in exercise tolerance. What if the magnitude of afferent feedback from the periphery was proportional to peripheral markers of impending physiological failure, and it was this peripheral response signal that governs the CNS response? Isn't this the antithesis of a central neural dominated theory like that of the CGM?

There is also an additional conundrum with the CGM. If motor unit recruitment is the focal piece of evidence to ascertain the operation of the central neural processing, and given that motor unit recruitment is a neurological event, how can there be any means to refute the presence of the CGM in any model of exercise tolerance? The only way to change motor unit recruitment is to alter neural processing. Eventually all forms of exercise intolerance is expressed through a diminished motor unit recruitment. As such the CGM is in fact unfalsifiable in the current wording, and therefore from a Popperian lens is pseudoscientific.

'Normal science', as introduced by Kuhn and criticised by Popper, is characterised by the premature acceptance of a theory due to inadequate critical enquiry. Instead, researchers pursue studies based on further applying the theory, or to intentionally further prove the theory. Based on Popper's perspective, you cannot prove a theory from intentionally designed research. Such research is more likely to apply bias to the research design, data analyses and interpretations.

One cannot peruse the research on the neural control of exercise tolerance since Noakes proposed the CGM without thinking back to the words of Popper where such work was labelled as pseudoscience. It is pseudoscience because it is too easy to design research based on the intent to prove something, and as such, force a desired result. Such research has the potential to direct knowledge away from the ultimate truth. If researchers want to support the CGM, then they need to vehemently attempt to disprove it, and show conclusively that such attempts at falsification continue to fail.

\section{Concerns about specialisation}

As an academic scientist initially educated within a 4year physical education undergraduate degree, followed by postgraduate education in the USA consisting of a Master's in Exercise and Sport Science and Cardiac Rehabilitation, followed by a PhD in Human Bioenergetics, it is reasonable to conclude that I have a broad education and research training. Such formal education has occurred across the basic and applied sciences spanning disciplines as diverse as organic chemistry, biochemistry, physics, biomechanics, biophysics, research methods, statistics, all physiological systems, pharmacology, molecular biology, health behaviour, computer programming and so on.

Throughout my career it has become increasingly evident that research of physiology, and especially exercise physiology, is handicapped by the subconscious bias of too many researchers to establish the single most important physiological explanation for a variety of topics of interest. I fear that this behaviour is spawned by our increasingly obvious trend of overspecialisation. Such a fear is a daunting reality when reading the following words from Popper's Preface, ${ }^{7}$ first published in 1938 !

\footnotetext{
'...only a revival of interest in these riddles [man's knowledge of the world in which he lives] can save the sciences and philosophy from narrow specialization and from an obscurantist faith in the expert's special skill and in his personal knowledge and authority; a faith that so well fits our 'post-rationalist' and 'postcritical' age, proudly dedicated to the destruction of the tradition of rational philosophy, and of rational thought itself. (p23)
}

Shouldn't the most important theories involving human physiology be integrative, not exclusionary to other physiological regulatory systems? I wonder whether the most serious obstacle facing the physiological sciences, and for that matter, all applications of science, is our modern rigid educational and training emphasis on specialisation! In this context, the evolution of the CGM to a more integrative model is encouraging. 
Nevertheless, I am concerned that the CGM has morphed into a theory of everything such that any organism with a brain and extended CNS is presumed to adhere to it. I was excited when I first read the manuscript from Lambert et $a l^{22}$ as I contemplated evidence of an awareness of the need for a model of multidisciplinary inclusion. However, there was still evidence of bias in support of the original CGM in this work. For example, Lambert et al proposed a new model involving different (multiple) physiological systems, but then stated 'evidence for central programming'. Furthermore, these authors advocated the presence and function of a new brain location, which they referred to as the 'teleoanticipatory central nervous system', but provide no empirical evidence of this centre.

Have they simply created this name because it aligned well with their model? If so, then such a development is pseudoscience.

Given the prior explanations of the errors of the CGM, I fear that all Noakes has accomplished is to replace one bias towards understanding exercise tolerance, that of cardiopulmonary limitations, with another bias, that of CNS control. I also fear that unless scientific enquiry begins to move from a paradigm of simplicity towards one of multidisciplinary complexity through integrative models, it will be a long time until we actually do witness a paradigm shift to a real scientific revolution of physiological understanding.

\section{Publication biases in editorial peer review}

This journal has been supportive of Noakes' commentaries on the CGM. ${ }^{15}$ 20-22 2426 This has been refreshing to see, for many scientific journals remain closed minded to threats to current dogmas. Nevertheless, any manuscript that eventually receives acceptance for publication in peer-reviewed journals must undergo peer review. I am not sure how to interpret the content of the journal within the acknowledgements of Pires' manuscript, ${ }^{1}$ where it is described that the manuscript underwent a non-commissioned external review. What does this mean? This is relevant, as I fail to comprehend how a blinded, impartial peer review would allow Pires' use and misinterpretation of citation numerics. Further, I do not see the value of a manuscript that only presents one side, a supportive side, of a controversial theory. Such journal conduct is inconsistent to the tenants of falsification, where quality scientific discourse would be to present attempts to repudiate a theory. In fact, I would go even further on this issue. I think it is the requirement of a scientist to be the most energetic ambassador of attempts to oppose their own theory. In this context, there would be no greater achievement in science than for a scientist to propose a new theory, and then be the one to most convincingly disprove it. The Popperian view would have all scientists be their own greatest critics.
I am also disturbed by the unequal presentation of content on the CGM that the journal has shown over the last 16 years. The task of falsification requires deliberate support by scientific journals for evidence from all views of a theory. If the journal wishes to support critical and creative scientific thinking supported by empiricism, then all interpretations need to be supported. As such, empirically supported criticisms of the CGM should be just as welcomed as manuscripts that support it, if not more so based on the tenant of falsification. Simply because the CGM may have revealed and removed a bias does not mean this alternate view, in any of its prior versions, is closer to the truth! Balanced, unbiased, critical commentary is essential and must continue if there is to be further progress on understanding the determinants to limitations of exercise tolerance. Scholarly journals are essential to this process in their role of fulfilling the dissemination component of the scientific method. Conversely, if scholarly journals fail in this endeavour, they inflict ripples of bias as feedback through the entire scientific method, exacerbating the incidence and severity of scientific dysfunction: pseudoscience.

\section{What needs to be done}

It is a shame that Noakes' brilliant critical enquiry of the physiology of constraints to exercise tolerance has ended in the absence of a well-worded theory. It is also confusing to see how the wording of the CGM has changed so dramatically across a 10-year span and remained so incompletely communicated despite a wealth of scholarly publication across this time interval.

Based on the prior content, it is reasonable to request the following from Noakes:

1. to change the name of the CGM to better reflect the latest iteration; specifically, the model should not reflect any connection to a central neural governor

2. to express the CGM as a theory, and in so doing be far more concise and adherent to the need to provide clear content to allow researchers to identify aspects of the theory that offer prediction of outcomes that can be used to develop research questions and methods that support falsification

3 . to remove all teleological terminology within the theory.

For researchers who have chosen to express support of the CGM through commentary and research scholarship, it would be more beneficial to science if they were to adhere to the following changes:

1. to develop research to critically challenge the theory resulting from the CGM and attempt to falsify it

2. to improve their efforts at the item above, such researchers need to invest effort to remove their personal biases, allowing themselves to seek and detect additional limitations of the model or theory and design research to test such limitations. 
For scholarly journals, regardless of the area of emphasis, there is a need to restructure their journal format, as well as editorial-peer review system, to promote and nurture a more critical process of research enquiry based on the process of falsification.

\section{CONCLUSIONS}

Science is a process, continually improving, hardly ever pursued in accordance with the philosophical ideals of its historical or contemporary development, and not a set of disciplines. Too many contemporary scientists do not understand the critical tenant of falsification embedded within the scientific method, and consequently this important feature of science has progressively become eroded from scientific practice. This is especially true for the exercise physiology and related disciplines of exercise science and each of basic, applied and clinical physiology. An understanding of the historical enlightenment provided by Kuhn's contributions to science provides evidence of this deterioration, and that such incorrect function, which Kuhn labelled 'normal science, ${ }^{9}{ }^{10}$ is far from normal. ${ }^{11}$

The CGM is a good example for how improper scientific function can lead to erroneous scientific processes and compromise data interpretations and conclusions. Unless a model is clearly expressed as a theory, and the theory is well worded to adhere to falsification (that from the wording of the theory components of the theory can be experimentally challenged and/or offer prediction of outcomes), future research of the topics at question loses structure and relevance. Furthermore, if such future research of the theory is conducted based on an intention of support rather than falsification, tremendous bias can be inserted into the research paradigm. The consequences of such scientific dysfunction are the generation of false knowledge, with subsequent delays in discovery along a path to the truth.

Karl Popper's critical evaluation of Kuhn's paradigm shifts, scientific revolutions and 'normal' science has important implications to exercise physiology. Until the basic, applied and clinical exercise physiology research fields adopt a more critical approach to the scientific method, supported by journal attributes that support the tenant of falsifiability, there will be constrained progress towards new knowledge. As such, exercise physiology would remain stagnant within Kuhn's 'normal science' phase, ${ }^{6}{ }^{7}$ which based on Popper's view would indeed be a consequence 'to be sorry for'. ${ }^{4}$

\section{Competing interests None declared.}

Provenance and peer review Not commissioned; externally peer reviewed.

Open Access This is an Open Access article distributed in accordance with the Creative Commons Attribution Non Commercial (CC BY-NC 4.0) license,

\section{Summary box}

The discipline of exercise physiology is not confined to exercise science. For more than 100 years, research of the physiological responses to exercise has been pursued by metabolic biochemists, specialist physiologists (eg, cardiovascular, pulmonary, neural, endocrine, muscle, bone and so on), clinicians (cardiologists, pulmonologists, physiotherapists, nutritionists, oncologists, epidemiologists and so on) and of course exercise scientists. The rich multidisciplinary input and applications of exercise physiology have been a major factor in the rapid growth of knowledge, and as with all disciplines of science no topic is owned by any one discipline.

- Thomas Kuhn's (1922-1996) textbook 'The structure of scientific revolutions', first published in 1962, was not written with the intent to detail how science should function. Kuhn wrote this text based on his belief that a review of the historical development of science would reveal important features about science that an idealistic philosophical platform would not.

- Popper's criterion of demarcation (that which separates pseudoscience from science) is the tenant of falsification. As such, scientists need to understand that the most effective way to prove a theory or specific knowledge is to demonstrate the inability to falsify it.

- Tim Noakes' central governor model is a good example of a construct that does not have a well-worded theory, and as such deviates from the tenant of falsification. Furthermore, research conducted with the intent to prove rather than disprove the theory contributes little to new knowledge and can condemn such research enquiry to the label of pseudoscience.

- The physiological sciences, and perhaps all of science, need to adhere more to the fundamental philosophical tenants of the scientific method. The peer-reviewed scholarly journals are a pivotal component of this system, and they must also change to be more supportive of constructive critical enquiry of presumably accepted theories and knowledge.

which permits others to distribute, remix, adapt, build upon this work noncommercially, and license their derivative works on different terms, provided the original work is properly cited and the use is non-commercial. See: http:// creativecommons.org/licenses/by-nc/4.0/

(C) Article author(s) (or their employer(s) unless otherwise stated in the text of the article) 2017. All rights reserved. No commercial use is permitted unless otherwise expressly granted.

\section{REFERENCES}

1. Pires FO. Thomas Kuhn's 'Structure of Scientific Revolutions' applied to exercise science paradigm shifts: example including the Central Governor Model. Br J Sports Med 2013;47:721-2.

2. Noakes TD. Challenging beliefs: ex Africa semper aliquid novi. Medicine \&amp Science in Sports \&amp Exercise 1997;29:571-90.

3. Katch V. Burden of disproof. Med Sci Sports Exerc 1986;18:593-5.

4. Robergs RA, Landwehr R. The Surprising history of the "HRmax=220-Age" Equation. JEPonline 2002;5:1-10.

5. Robergs RA, Ghiasvand F, Parker D. Biochemistry of exerciseinduced metabolic acidosis. Am J Physiol Regul Integr Comp Physiol 2004;287:R502-R516.

6. Robergs RA. A critical review of the history of low- to moderateintensity steady-state VO2 kinetics. Sports Med 2014;44:641-53.

7. Popper KR. The logic of scientific discovery. Connecticut: Mansfield Centre:Martino Publishing, 2014:27-111.

8. Tipton CM. Exercise physiology, Part II: A contemporary historical perspective. In: Massengale JD, Swanson RA, eds. The history of 
exercise and sports science. Champaign, IL: Human Kinetics, 1997:396-438.

9. Kuhn T. The structure of scientific revolutions. Chicago,IL: University of Chicago Press, 1962:xxxix-172.

10. Kuhn TS. Logic of discovery or psychology of research? In: Lakatos I, Musgrave A, eds. Criticisms and the growth of knowledge. cambridge IRP: University Press, 1995:1-23.

11. Popper KR. Normal science and its dangers. In: Lakatos I, Musgrave A, eds. Criticisms and the growth of knowledge. Cambridge,IRP: University Press, 1995:51-8.

12. Okasha S. Philosophy of science: a very short introduction. New York: Oxford University Press, 2000:4-20.

13. Noakes TD. Physiological models to understand exercise fatigue and the adaptations that predict or enhance athletic performance. Scand J Med Sci Sports 2000;10:123-45.

14. Noakes TD, Peltonen JE, Rusko HK. Evidence that a central governor regulates exercise performance during acute hypoxia and hyperoxia. J Exp Biol 2001;204:3225-34.

15. Noakes TD, St Clair Gibson A, Lambert EV. From catastrophe to complexity: a novel model of integrative central neural regulation of effort and fatigue during exercise in humans. Br J Sports Med 2004;38:511-4.

16. Farina D, Merletti R, Enoka RM. The extraction of neural strategies from the surface EMG. J App/ Physiol 2004;96:1486-95.

17. Farina D. Counterpoint: spectral properties of the surface EMG do not provide information about motor unit recruitment and muscle fiber type. J Appl Physiol 2008;105:1673-4.

18. Farina D. Last word on point:counterpoint: spectral properties of the surface EMG can characterize/do not provide information about motor unit recruitment and muscle fiber type. J Appl Physiol 2008;105:1683.

19. von Tscharner V, Nigg BM. Point: spectral properties of the surface EMG can characterize/do not provide information about motor unit recruitment strategies and muscle fiber type. J Appl Physiol 2008;105:1671-3.

20. St Clair Gibson A, Noakes TD. Evidence for complex system integration and dynamic neural regulation of skeletal muscle recruitment during exercise in humans. $\mathrm{Br} J$ Sports Med 2004;38:797-806.

21. Noakes TD, St Clair Gibson A, Lambert EV, et al. From catastrophe to complexity: a novel model of integrative central neural regulation of effort and fatigue during exercise in humans: summary and conclusions. Br J Sports Med 2005;39:120-4.

22. Lambert EV, St Clair Gibson A, Noakes TD, et al. Complex systems model of fatigue: integrative homoeostatic control of peripheral physiological systems during exercise in humans. Br J Sports Med 2005;39:52-62.

23. Noakes TD. The central governor model of exercise regulation applied to the marathon. Sports Med 2007;37:374-7.

24. Noakes TD. Testing for maximum oxygen consumption has produced a brainless model of human exercise performance. $\mathrm{Br} J$ Sports Med 2008;42:551-5.

25. Noakes TD. Time to move beyond a brainless exercise physiology: the evidence for complex regulation of human exercise performance. Appl Physiol Nutr Metab 2011;36:23-35.

26. Noakes TD. The Central Governor Model in 2012: eight new papers deepen our understanding of the regulation of human exercise performance. Br J Sports Med 2012;46:1-3.

27. Saltin B, Rådegran G, Koskolou MD, et al. Skeletal muscle blood flow in humans and its regulation during exercise. Acta Physiol Scand 1998;162:421-36.

28. Joyner MJ, Casey DP. Regulation of increased blood flow (hyperemia) to muscles during exercise: a hierarchy of competing physiological needs. Physiol Rev 2015;95:549-601.

29. Richardson RS, Noyszewski EA, Kendrick KF, et al. Myoglobin O desaturation during exercise. evidence of limited $\mathrm{O}_{2}$ transport. J Clin Invest 1995;96:1916-26.

30. Bassett DR, Howley ET. Limiting factors for maximum oxygen uptake and determinants of endurance performance. Med Sci Sports Exerc 2000;32:70-84.

31. Levine BD. VO2max: what do we know, and what do we still need to know? J Physiol 2008;586:25-34.

32. Shephard RJ. Is it time to retire the 'central governor'? Sports Med 2009;39:709-21. 